\title{
Rosai-Dorfman Disease (Sinus Histiocytosis with Massive Lymphadenopathy) With Cutaneous and Ocular Involvement: A Case Report
}

\author{
H. C. WONG, ${ }^{*}$ J. E. WRIGHT* AND J. HARRY† \\ London and Birmingham
}

\begin{abstract}
Summary
This case report presents both the clinical and the histopathological features of the oldest patient on record with ophthalmic manifestations of Rosai-Dorfman Disease. An unusual feature, which has not been previously reported, is involvement of the cornea with subsequent perforation of the globe. The absence of lymphadenopathy is discussed.
\end{abstract}

Rosai and Dorfman described sinus histiocytosis with massive lymphadenopathy (SHML) in $1969,{ }^{1}$ and ten years later, together with Foucar, ${ }^{2}$ reported ophthalmic involvement in 13 of 113 cases of SHML (11.5\%). The cause of the disease remains unknown. Our case is reported for the following reasons:

(1) At presentation, there was cutaneous and ophthalmic involvement only; no lymphadenopathy was detectable clinically.

(2) The patient is the oldest on record with ophthalmic manifestations.

(3) The globe was involved to such an extent that partial exenteration was necessary.

\section{History}

A 40 year old West African male had noted, for 18 months, four painless swellings on his face; there were also swellings on both ears, the left shoulder, and chest. Vision in the left eye had been lost for four months. More recently, he had complained of nasal congestion but his general health otherwise was good.

\section{Physical examination}

On examination, visual acuity was $6 / 6$ in the right eye and perception of light in the left eye. Facial lesions were noted (Fig. 1); the largest, involving the left lower lid, had infiltrated the inferior three quarters of the cornea, thus totally obliterating the visual axis, and at the junction of the lesion with the cornea there was a descemetocele. General clinical examination revealed other lesions on the left shoulder and the chest (Fig. 2). No other obvious abnormality was found; he was apyrexial, and there was neither lymphadenopathy nor hepatosplenomegaly.

\section{Investigations}

(1) Haematology

(a) Full blood count: normal; no leucocytosis

(b) Haemoglobin electrophoresis: heterozygote sickle cell disease $(\mathrm{A} / \mathrm{S})$.

(2) Immunology

(a) C-Reactive protein: raised $(9.01 \mathrm{mgm} /$ litre-normal<6).

(b) IgG: slightly raised IgA and IgM: within normal limits Serum electrophoresis: no monoclonal band 


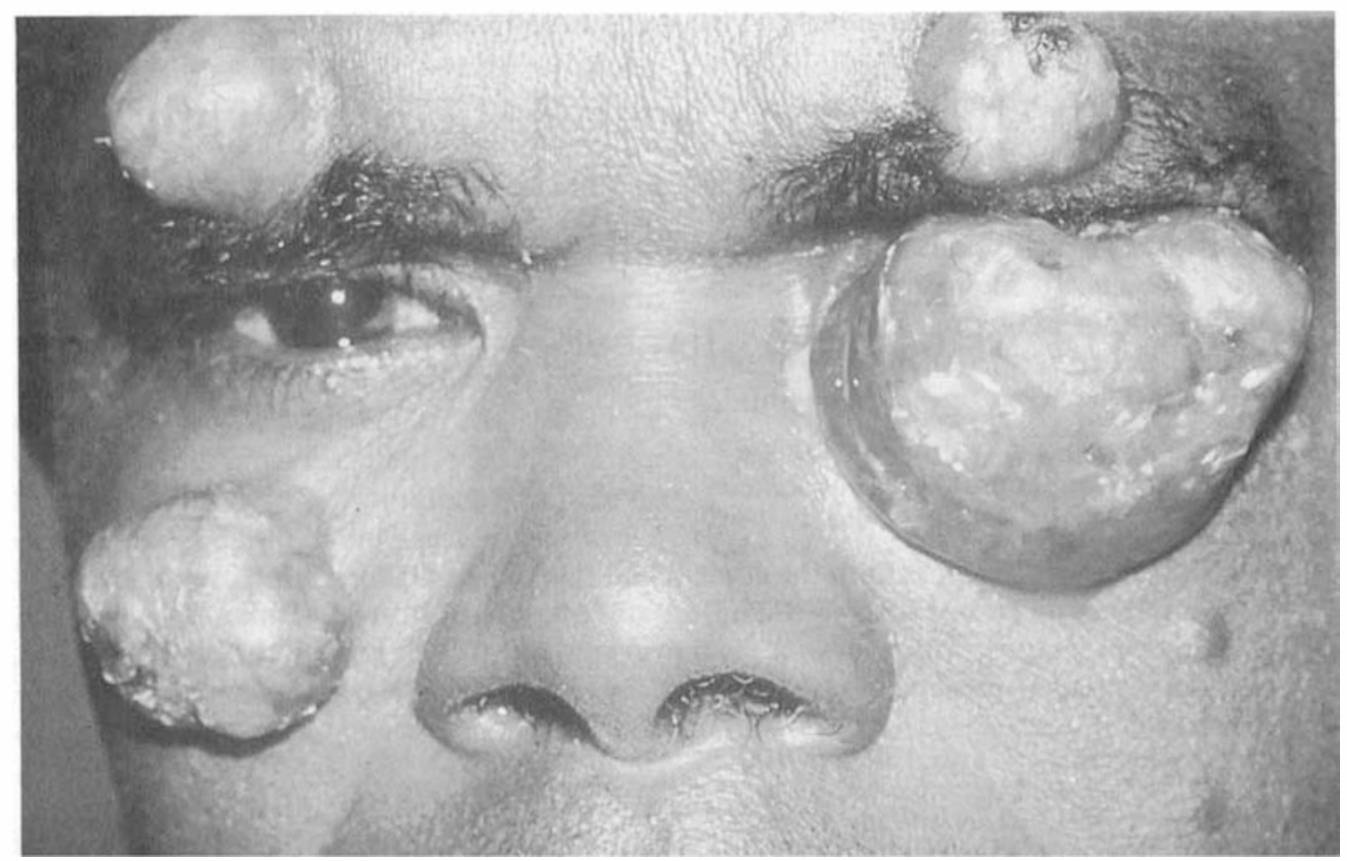

Fig. 1. Tumour affecting both eyebrows, the right cheek and the left lower lid.

(b) Autoantibody screen: negative

(3) Computerised axial tomography (Fig 3)

A soft tissue mass involved the left lower lid, but the orbital contents were apparently normal; the nasal septum was thickened.

\section{Biopsy}

The lesion on the left eyebrow was removed and referred for histopathological study. Sections showed, within the dermis, marked fibrosis and a dense cellular infiltrate consisting of lymphocytes, plasma cells and histiocytes (Fig. 4(a) and (b)). The latter were arranged in large groups and showed conspicuous nuclei, prominent nucleoli, foamy cytoplasm and cytophagocytosis (Fig. 5) On the basis of these features a diagnosis of SHML was made.

\section{Treatment}

A single dose of eight gray was given to the lesion on the right eyebrow, and this, over the following week, regressed to half of its original size. Subsequently each of the other lesions received 18 gray in six sessions; these, however, did not regress, and the one on the right cheek actually increased in size. By one month later, the lesion on the left lower lid had increased to such a size, that the globe had perforated at the site of the descemetocele. At this time the remaining eyebrow lesion and also the tumour on the right cheek were removed, and a partial left exenteration was performed together with excision of the left lid. There was no clear plane of cleavage between the lid and normal tissue at operation; there was profuse haemorrhage and it became necessary to apply extensive diathermy.

\section{Pathology}

Sections of the lesions from the eyebrow and the cheek revealed the histopathological features of SHML as previously described. The exenterated specimen showed to one side of the globe, a large extraocular mass merging imperceptibly with the cornea and sclera. Microscopy of this mass also revealed the histopathological features of SHML, but with a greater degree of fibrosis than that noted in the other lesions. There was considerable surface inflammation and the unaffected cornea was thinned, scarred and vascularised; the site of 


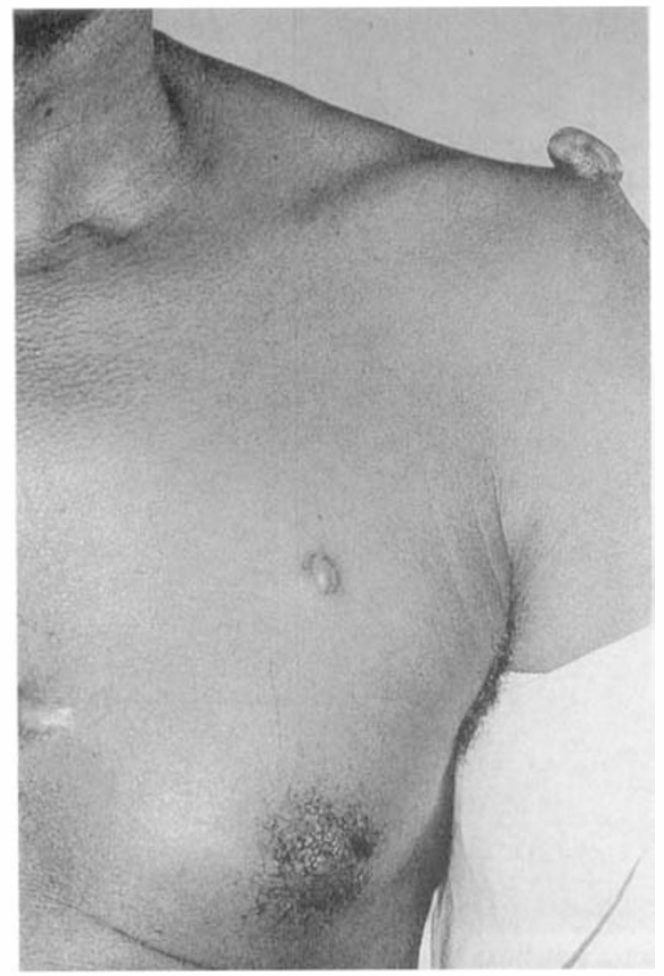

Fig. 2. Tumours affecting the left shoulder and the chest

the descemetocele was seen and there was evidence of previous perforation. The eye also exhibited rubeosis iridis and the filtration angle on the side of the extraocular mass was occluded; in addition, the ciliary body was infiltrated by similar lymphoid tissue to that seen outside the eye.

\section{Discussion}

SHML presents typically in Negro children with gross cervical lymphadenopathy and fever; investigations often show leucocytosis with neutrophilia, an elevated erythrocyte sedimentation rate and hypergammaglobulinaemia. Extranodal sites including the orbit, the eyelids, the upper respiratory tract, the salivary glands, skin, bone and the testes are involved in $28 \%$ of cases. SHML may mimic a malignant disease and presentation is often startling, but contrary to its clinical appearance, its course is relatively benign. The lesions, however, may take many years to resolve, but during this time the patients remain well systemically. In the review by Foucar et al. , ${ }^{2}$ only one of thirteen patients with ophthalmic SHML died; this patient, thought to have malignant histiocytosis, was treated with antibiotics, corticosteroids, chemotherapy and radiotherapy, and finally succumbed to multiple episodes of bronchopneumonia. Of the remaining twelve, after the onset of ophthalmic disease, four had no significant evidence of SHML three to 11 years later, whereas four showed persistent ophthalmic and/or lymph node involvement when seen between two and a half and 19 years later. The remaining cases were either lost to follow-up or the period of follow-up was too short to be of significance. Neither surgery, chemotherapy nor radiotherapy consistently led to remission.

Our case is atypical. At 40 years of age, the patient is the oldest on record with ophthalmic manifestations of SHML and the involvement of the cornea which led to perforation of the globe has not been reported previously. The mechanism of the corneal perforation was due presumably to the inability of the tear film to wet the raised lesion and the adjacent normal cornea; thus corneal thinning developed progressively. Although the lesion on the right eyebrow regressed with radiotherapy, the remaining lesions responded poorly and underlined the relative aggressive nature of the disease. This discrepancy of response to radiotherapy may have been due to the fortuitous coincidence of spontaneous regression of the lesion on the right eyebrow with the course of radiotherapy.

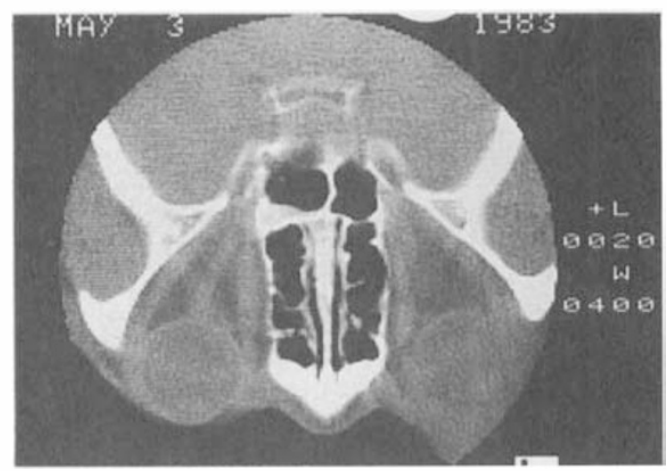

Fig. 3. CT scan of the orbits showing a soft tissue mass anterior to the left globe; the nasal septum is thickened. 
348.

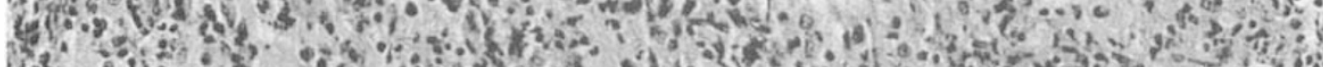

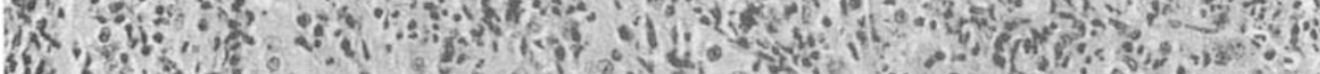

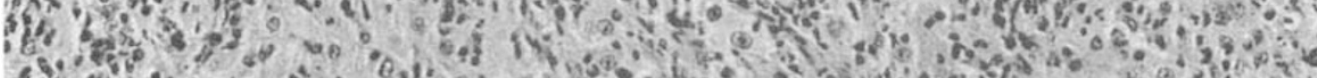

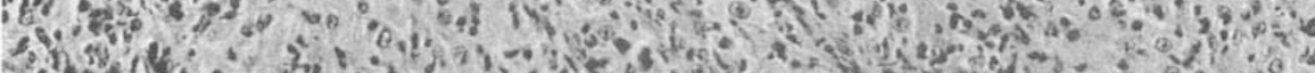

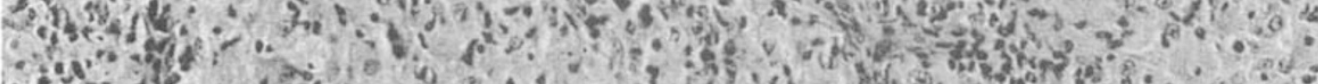

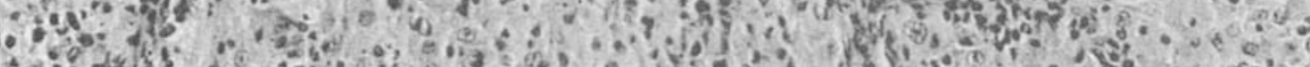

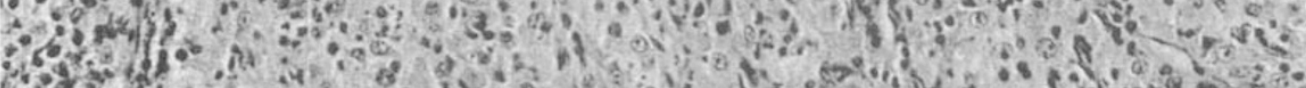
6.0.7.

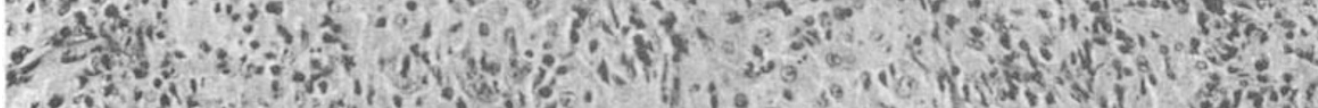

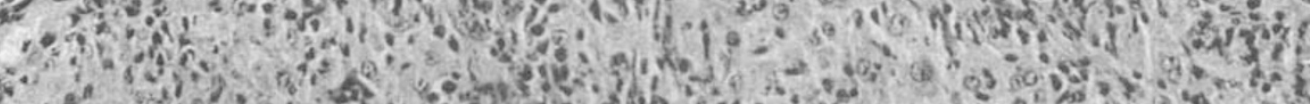
E. If

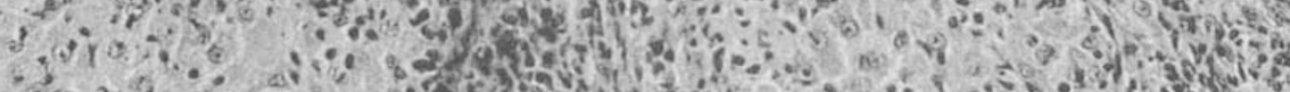

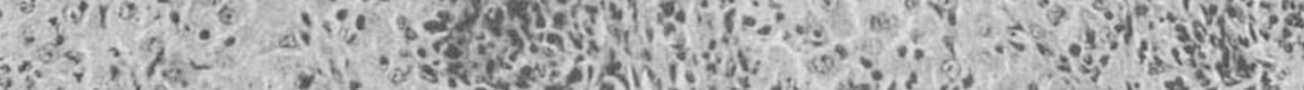

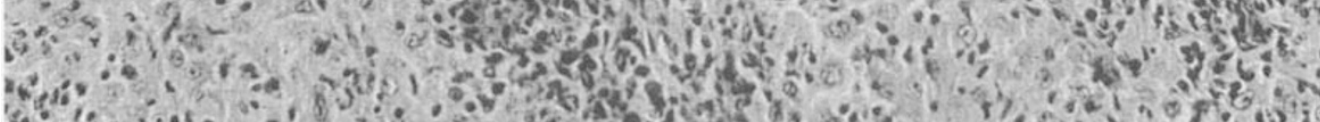

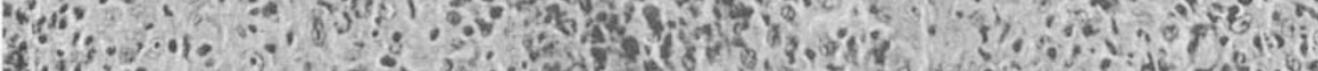

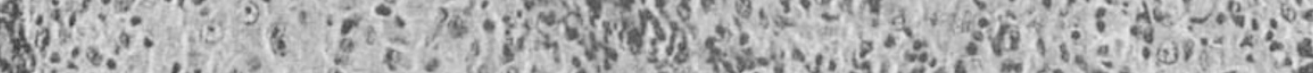

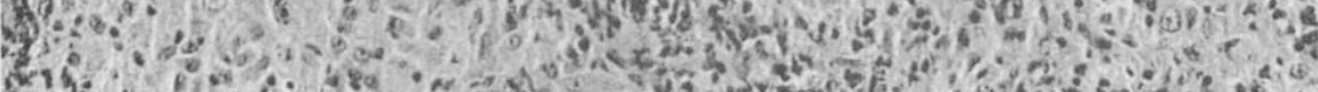

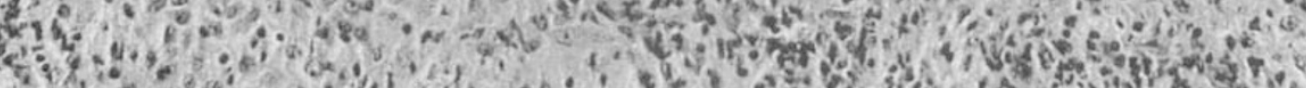

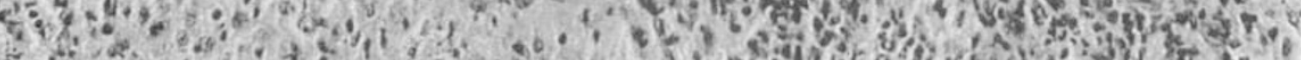

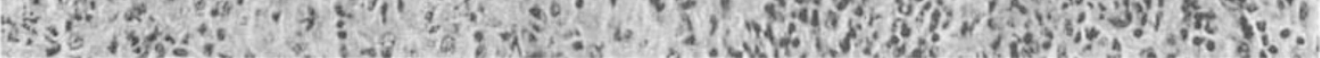

Fig. 4 (a) and (b). There is fibrosis and a mixed cell population; histiocytes are particularly well seen in (b) Heamatoxylin and eosin $\times 200$ 


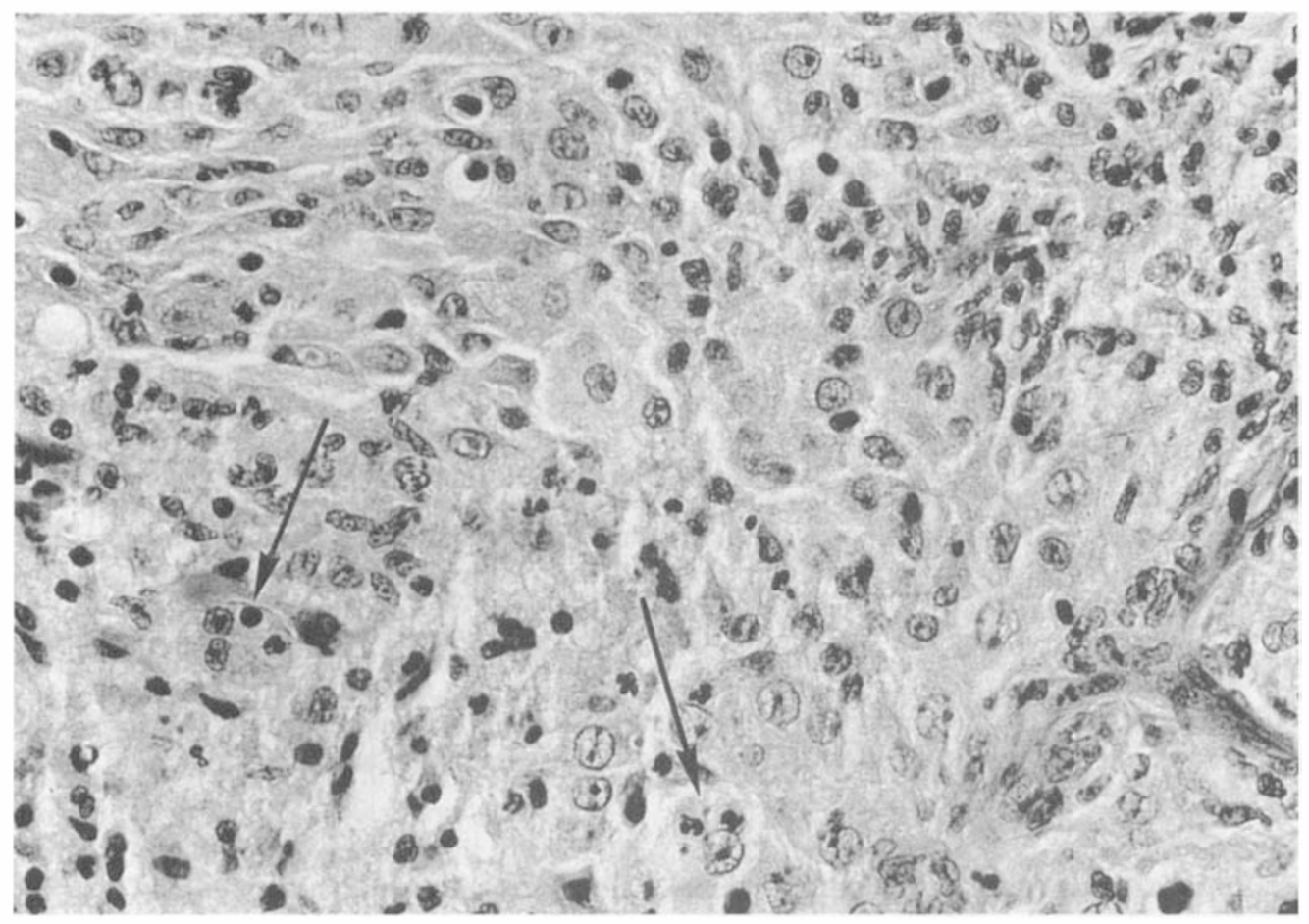

Fig. 5. An area showing histiocytes exhibiting cytophagocytosis (arrowed) Haematoxylin and eosin $\times 550$

The clinical differential diagnosis of SHML includes a spectrum of inflammatory, and both benign and malignant neoplastic conditions, but because our case had no lymphadenopathy, a clinical diagnosis of SHML was not considered. Histopathological study, however, revealed the diagnosis. Microscopy shows the dominant feature to be the presence of large histiocytes with vesicular nuclei, prominent nucleoli and abundant eosinophilic cytoplasm. Thereis cytophagocytosis and the histiocytes may contain red and white blood cells, and plasma cells. In classic SHML these histiocytes are seen in abundance in the lymph nodes of the cervical chain; less commonly, however, they aggregate in extranodal sites and, as in our case, there may be no lymphadenopathy. In the present state of knowledge such an entity is probably best designated Rosai-Dorfman Disease.
For technical help our thanks are due to Mr. I. Rhodes and Miss R. Hart of the Institute of Ophthalmology, London, and to Mr. R. Hickton of the Birmingham and Midland Eye Hospital. For assistance with the photomicrographs we are grateful to Mr. I. J. Bradley of the Birmingham and Midland Eye Hospital. Our thanks also to Dr. A. G. Stansfeld, Reader Emeritus in Pathology at St. Bartholomew's Hospital, London, to Dr. R. F. Dorfman, Laboratory of Surgical Pathology, Stanford University Medical Centre, California, to Dr. J. M. Henk, Consultant in Radiology and Oncology, The Royal Marsden Hospital, London, and to Dr. G. Lloyd, Consultant Radiologist, Moorfields Eye Hospital, London. For secretarial work we thank Mrs. A. Minto of the Birmingham and Midland Eye Hospital.

\section{References}

${ }^{1}$ Rosai J and Dorfman RF: Sinus Histiocytosis with Massive Lymphadenopathy. A newly recognised benign clinicopathological entity. Arch Pathol 1969, 87: 63-70.

${ }^{2}$ Foucar E, Rosai J, Dorfman RF: The Ophthalmic mainfestations of Sinus Histiocytosis with Massive Lymphadenopathy. Am J Ophthalmol 1979, 87: 354-67. 